FACTORES CLAVE QUE INFLUYEN EN UNA MUESTRA GASTRONÓMICA

\title{
FACTORES CLAVE QUE INFLUYEN EN UNA MUESTRA GASTRONÓMICA
}

\author{
KEY INFLUENCING FACTORS GASTRONOMIC SAMPLE
}

Gabriela Velasco Rodríguez*, Valeria Cano Castillo**

*Doctorado en Ciencias de la Administración. Universidad Autónoma de Ciudad Juárez.

**Estudiante de la Licenciatura en Turismo. Universidad Autónoma de Ciudad Juárez.

Dirección para recibir correspondencia: gvelasco@uacj.mx

Fecha de recibido: 12 de septiembre de 2018

Fecha de aceptación: 26 de noviembre de 2018 
FACTORES CLAVE QUE INFLUYEN EN UNA MUESTRA GASTRONÓMICA

\section{RESUMEN}

OBJETIVO: Analizar los factores clave que influyen en una muestra gastronómica para determinar el perfil de los participantes.

MATERIAL Y MÉTODO: Se trata de una investigación no experimental, transversal y descriptiva, aplicada a los participantes en una muestra gastronómica en la Universidad Autónoma de Ciudad Juárez. Se utiliza un cuestionario de 14 preguntas. El análisis de datos, se realizó por medio de estadística descriptiva.

RESULTADOS: Los factores culturales, sociodemográficos, de personalidad y de experiencia que influyen en una muestra gastronómica permiten visualizar el perfil de los participantes. El perfil del participante es principalmente mujeres que oscilan entre los 20 y 29 años. Se obtiene que el sabor más conocido es dulce, de los ingredientes que se usan con mayor frecuencia, son: sal y limón, asimismo la principal técnica de preparación es hervir. Finalmente, la importancia de una muestra gastronómica radica en mejorar la cultura, conocer las diferencias y aprender.

CONCLUSIONES: Los factores clave de una muestra gastronómica, permite la caracterización del perfil del participante, implicando contar con una herramienta que pueda contribuir a la materialización del marketing gastronómico.

PALABRAS CLAVE: Factores. Muestra. Gastronómica. Estudiantes.

\section{ABSTRACT}

OBJECTIVE: To analyze the key factors influencing a gastronomic sample to determine the participants' profile.

MATERIAL AND METHODS: This is a non-experimental, cross-sectional descriptive study, carried out with participants in a gastronomic sample at Universidad Autonoma de Ciudad Juarez. A questionnaire of 14 questions was administered. Data analysis was performed by using descriptive statistics.

RESULTS: Cultural, socio-demographic, personality and experience factors that influence during a gastronomic sample, allow the observation of the participant's profile. The main VELASCO-RODRÍGUEZ G., CANO-CASTILLO V. 
FACTORES CLAVE QUE INFLUYEN EN UNA MUESTRA GASTRONÓMICA

participant profile is female, aged from 20 to 29 years. The information obtained is that the bestknown flavor is sweet, the most common ingredients used, are: salt and lemon; furthermore, boiling is the main cooking technique. Finally, the relevance of a gastronomic sample is to improve the culture, know about cultural differences and learn from them.

CONCLUSIONS: The key factors of a gastronomic sample allow the contrast of the participants' profile, which implies having a specific instrument that can also contribute to the history in the making of gastronomic marketing.

KEYWORDS: Factors. Sample. Gastronomic. Students.

\section{INTRODUCCIÓN}

Una parte fundamental del marketing gastronómico, se da principalmente en el desarrollo de las bases gastronómicas; es decir, cómo es que se dan a conocer las bases de la gastronomía que constituyen los platillos para cada cultura, cómo lo perciben las otras culturas, cómo se realiza cada platillo, qué diferencias existen entre cada una de ellas y qué factores las conforman.

Acorde con la esencia del significado de "marketing gastronómico", la gastronomía es el estudio de la relación entre cultura y alimento (De Alva, 2012, p. 6), además, se considera arte culinario focalizado en sus vertientes de deleite y creatividad (Montes, Lloret, \& Lopez, 2009, p. 11).

En lo referente a marketing, es el arte de hacer converger las acciones de la empresa de cara a satisfacer mejor las necesidades de su clientela, en el marco de políticas coherentes que buscan optimizar la eficacia global de la empresa de cara a su mercado (Rochat, 2000, p. 92) citado en (Cabrera, 2013, p. 168).

Una de las formas en la que se puede observar la esencia del marketing gastronómico es a través de las muestras gastronómicas, donde se prepara un número variado de platillos y bebidas, y se dan a probar a distintos visitantes. Del tipo de muestras gastronómicas que se pueden dar, por su accesibilidad son las muestras universitarias son las que más se prestan para un estudio más detallado.

En lo referente a la muestra gastronómica, es reconocida por sus sabores, ingredientes y técnicas de preparación alusivo a los factores culturales con base a los factores 
FACTORES CLAVE QUE INFLUYEN EN UNA MUESTRA GASTRONÓMICA

sociodemográficos, donde a partir de la edad es posible determinar algunos hábitos y costumbres de compra, asimismo la variable sexo permite identificar las diferencias y hábitos de consumo por el estado civil determinado por el matrimonio e incide directamente en su costumbre de compra, ya sea por la escolaridad que se refiere al nivel de instrucción alcanzado como por la ocupación que puede ser utilizada como referencia de la actividad a la que se dedican las personas.

Con referencia a los factores de personalidad se pueden distinguir dos tipos, por un lado, el individuo que se define como aquel que se adapta rápidamente al cambio, o sea, es una persona neofilia, asimismo corresponde a la etapa en la que el acostumbramiento rápido a situaciones placenteras lleva a estar siempre dispuesto para la búsqueda de nuevos momentos de placer (Ordoñez, 2010, p. 64). Por otra parte, se encuentra cuando un individuo no le gusta experimentar cosas nuevas; es decir, es una persona con neofobia que significa miedo a lo nuevo (Bunge, 2011, p. 59).

En lo que respecta a los factores de experiencia, se consideran tres aspectos: primero es la importancia de la muestra gastronómica; segundo es la obtención de las recetas que depende del acceso a los medios impresos y/o digitales; por último, es lo que caracteriza al platillo y/o bebida; por ello, una vez elaborada la receta, los tres aspectos son importantes en cuanto al sabor, presentación y originalidad. Por lo anterior, se pretende analizar los factores clave antes mencionados como influyen en una muestra gastronómica para determinar el perfil de los participantes.

\section{MATERIAL Y MÉTODO}

Se trata de una investigación no experimental transversal descriptiva, aplicada a una población de visitantes como expositores que participaron en la XIII muestra gastronómica del programa académico de turismo, realizada el 27 de abril de 2017 en las instalaciones del Instituto de Ciencias Sociales y Administración en la Universidad Autónoma de Ciudad Juárez. Se elabora un cuestionario que comprende 14 preguntas distribuidas de la siguiente manera: tres preguntas para describir los factores culturales como son sabores, ingredientes y técnicas de preparación; seis preguntas que abordan las variables de los factores sociodemográficos; dos preguntas para representar los factores de personalidad; y las últimas tres preguntas se refieren a los factores de experiencia. En la tabla 1, se muestran las variables de estudio con su respectiva explicación. En el procedimiento para obtener la información de los factores clave, 
FACTORES CLAVE QUE INFLUYEN EN UNA MUESTRA GASTRONÓMICA

primero, se recaban y analizan los datos cualitativos para obtener categorías. Después, se utilizaron los resultados para construir un instrumento, donde se pueda concebir los ítems y escalas para cada una de las categorías y, posteriormente, se administra el instrumento a una muestra de una población. Finalmente, se realiza el análisis de los datos para obtener los resultados y se puede hacer inferencias al respecto.

\section{Tabla 1}

Variables de estudio

\begin{tabular}{|c|c|c|c|}
\hline Campo & Descripción & Tipo & Codificación \\
\hline \multicolumn{4}{|l|}{ Factores culturales } \\
\hline Sabores & Mencionar sabores & Texto & --- \\
\hline Ingredientes & Identificar ingredientes & Texto & --- \\
\hline Técnicas de preparación & Distinguir técnicas & Texto & --- \\
\hline \multicolumn{4}{|l|}{ Factores sociodemográficos } \\
\hline \multirow[t]{6}{*}{ Edad } & Rango de edad & Numérico & $1=15-19$ \\
\hline & & & $2=20-24$ \\
\hline & & & $3=25-29$ \\
\hline & & & $4=30-34$ \\
\hline & & & $5=35-39$ \\
\hline & & & $6=40$ y más \\
\hline \multirow[t]{2}{*}{ Sexo } & Seleccionar el sexo & Numérico & 1= Femenino \\
\hline & & & 2= Masculino \\
\hline \multirow[t]{5}{*}{ Estado civil } & Determinado por el & Numérico & 1= Soltero \\
\hline & matrimonio & & $2=$ Casado \\
\hline & & & 3= Viudo \\
\hline & & & 4= Divorciado \\
\hline & & & 5= Unión libre \\
\hline \multirow[t]{6}{*}{ Escolaridad } & Nivel educativo & Numérico & 1=Primaria \\
\hline & & & 2=Secundaria \\
\hline & & & $3=$ Preparatoria \\
\hline & & & 4= Universidad \\
\hline & & & 5= Postgrado \\
\hline & & & $6=$ Sin escolaridad \\
\hline \multirow[t]{2}{*}{ Ocupación } & Actividad a la que se & Texto & \\
\hline & dedica & & --- \\
\hline Ingreso familiar & Nivel adquisitivo & Numérico & Cantidad en pesos \\
\hline
\end{tabular}

VELASCO-RODRÍGUEZ G., CANO-CASTILLO V. 
FACTORES CLAVE QUE INFLUYEN EN UNA MUESTRA GASTRONÓMICA

\begin{tabular}{|c|c|c|c|}
\hline \multicolumn{4}{|l|}{ Factores de personalidad } \\
\hline $\begin{array}{l}\text { Soy una persona que se } \\
\text { adapta rápidamente al cambio }\end{array}$ & Adaptación & Numérico & $\begin{array}{l}1=\text { Sí } \\
2=\text { No }\end{array}$ \\
\hline $\begin{array}{l}\text { Soy una persona que no le } \\
\text { gusta experimentar cosas } \\
\text { nuevas }\end{array}$ & Tendencia & Numérico & $\begin{array}{l}1=\text { Sí } \\
2=\text { No }\end{array}$ \\
\hline \multicolumn{4}{|l|}{ Factores de experiencia } \\
\hline $\begin{array}{l}\text { Importancia de la muestra } \\
\text { gastronómica }\end{array}$ & Significado & Texto & --- \\
\hline Obtención de la receta & Lugar & Texto & --- \\
\hline Características de una receta & Distinción de una receta & Texto & --- \\
\hline
\end{tabular}

Fuente: Elaboración propia.

\section{RESULTADOS}

Los resultados se obtienen en la presente investigación brindan un amplio panorama de los factores clave que influyen en una muestra gastronómica que permite visualizar el perfil de los participantes; seguidamente, se puede encontrar los datos relevantes por cada uno de los factores.

En los factores culturales se puede apreciar los sabores, ingredientes y técnicas de preparación; los cuatro tipos de sabores más conocidos son dulces, salados, amargos y picosos; Otros mencionan, agrio y ácido. En relación con los cinco ingredientes que se usan con más frecuencia en los platillos son: sal, carne y pimienta, en cambio, limón y alcohol en las bebidas. En menor proporción, se encuentran azúcar, chile, pollo, pan, ajo, harina, aceite, pescado, cebolla y licor. Con respecto a las tres principales técnicas de preparación que les llama la atención, son: a la leña, al carbón y a la plancha, en los visitantes, por el contrario, los expositores mencionan hervir, freír y asar. Adicionalmente, marinado, al vapor, horneado, rostizado, shake, saltear, concasse, entre otros. A continuación, se presentan las distintas técnicas de preparación (ver figura 1). 
FACTORES CLAVE QUE INFLUYEN EN UNA MUESTRA GASTRONÓMICA

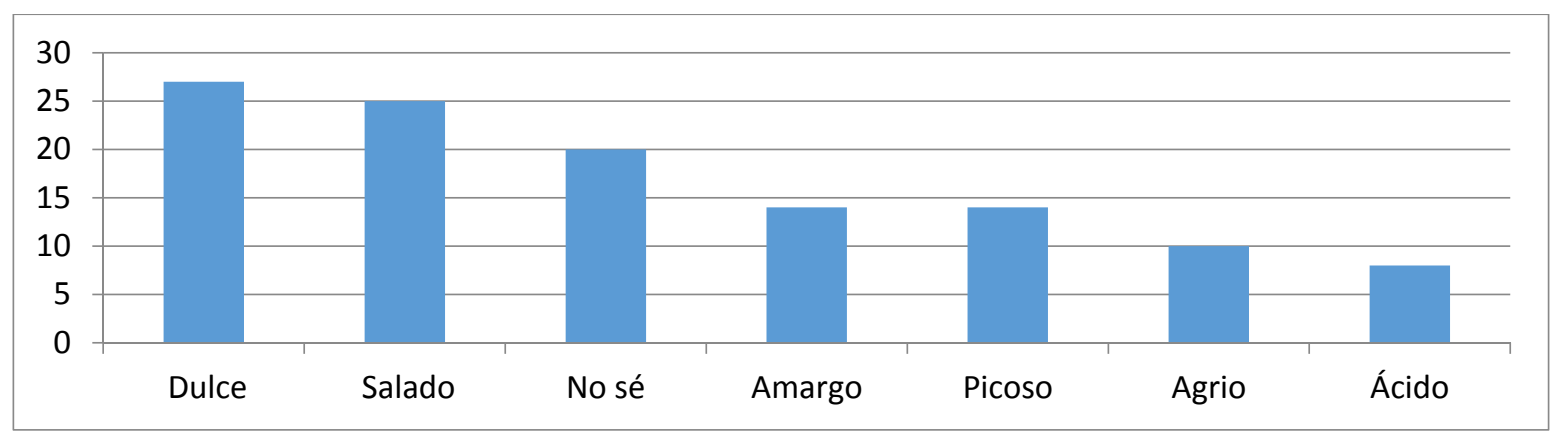

Figura 1. Técnicas de preparación.

Fuente: Elaboración propia.

En lo que corresponde a los factores sociodemográficos, la mayoría de los visitantes se encuentra en el rango de edades de los 25 a los 29 años, en cambio los expositores se encuentran entre los 20 y 24 años, donde 6 de cada 10 son del sexo femenino que se dedican a estudiar y la mayor parte son solteros. En la figura 2, se representa la proporción de participantes por sexo.

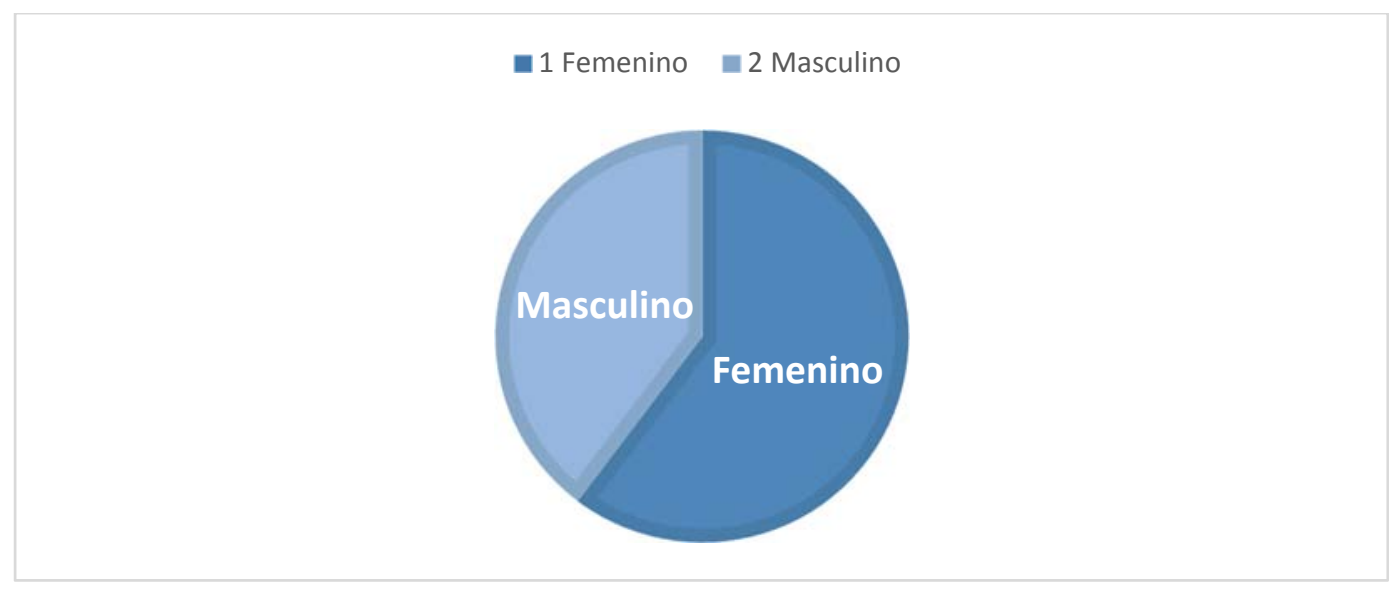

\section{Figura 2. Distribución por sexo.}

Fuente: Elaboración propia.

Respecto a los factores de personalidad, destaca que la generalidad de los visitantes no se adapta rápidamente al cambio, en oposición a los expositores les gusta experimentar cosas nuevas. 
FACTORES CLAVE QUE INFLUYEN EN UNA MUESTRA GASTRONÓMICA

Por otra parte, en los factores de experiencia, tanto los visitantes como expositores manifiestan que la importancia de una muestra gastronómica radica en mejorar la cultura, conocer diferencias y aprender. También, las obtenciones de las recetas son mediante internet, experimentación o del restaurante, considerando que lo más relevante de una receta es sabor, después, originalidad y, posteriormente, presentación (ver figura 3).

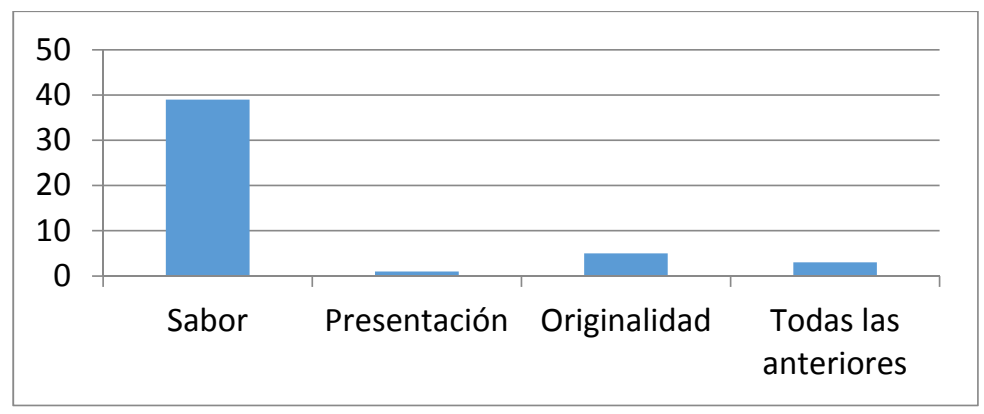

Figura 3. Variables de una receta.

Fuente: Elaboración propia.

\section{CONCLUSIONES}

Con base al objetivo de la presente investigación, en los factores clave que influyen en la muestra gastronómica, destaca una caracterización del perfil del participante que implica en primer término, el significado de la muestra gastronómica que radica en conocer las diferencias en los sabores, ingredientes y técnicas, donde la degustación se palpa a través del sabor como de la originalidad y de la presentación de platillos y bebidas que se ven influidos por las características sociodemográficas y de personalidad del participante, dentro del espacio de la muestra gastronómica, ya sea del visitante o del exponente.

Por lo anterior, permite a los interesados visualizar los factores que hay que considerar al realizar una muestra gastronómica, donde se pueda analizar las diferencias y similitudes en relación con cuatro factores clave, ya sean culturales, sociodemográficos, de personalidad, y experiencia, para valorar los significados de la esencia de una muestra gastronómica mediante el perfil de los participantes. En ese sentido, la aportación del estudio fue el diseño de una herramienta, cuya finalidad es contribuir a la medición de la materialización del marketing gastronómico. 
FACTORES CLAVE QUE INFLUYEN EN UNA MUESTRA GASTRONÓMICA

\section{REFERENCIAS BIBLIOGRÁFICAS}

Bunge, M. (2007). 100 Ideas: El libro para pensar y discutir en el cafe. Buenos Aires: Sudamericana.

Cabrera, S. (2013). Marketing gastronómico. En cuaderno 45 | Centro de Estudios en Diseño y Comunicación (2013). pp. 165-174, ISSN 1668-5229.

Gutierrez de Alba, C. I. (2012). Historia de la gastronomía. Estado de México: Red Tercer Milenio S.C.

Make, A., Lumbers, M., Eves, A., \& Chang, R. (2011). Factors influencing tourist food consumption. International Journal of Hospitality Management, 31(3), 928-936.

Mejía López, L. S., Mejía Castillo, S. E., \& Bravo Rodríguez, M. (2014). Tendencias gastronómicas: La encrucijada entre lo tradicional y lo innovador. Culinaria, Revista virtual especializada en gastronomía, p. 28.

Montes, E., Lloret, I., \& Lopez, M. A. (2009). Diseño y gestión de Cocinas. España: Diaz de Santo.

Oliveira, S. (ene/jun de 2011). La gastronomía como atractivo turístico primario de un destino. Estudios y perspectivas en turismo, 20(3), 738-752. Recuperado de: http://www.scielo.org.ar/scielo.php?script=sci_arttext\&pid=S1851-17322011000300012

Ordoñez, R. (2010). La ruta hacia la transformación. En R. Ordoñez, Cambio, Creatividad e Innovación, (pp. 64-65). Buenos Aires: Ediciones Granica.

Torres Rodríguez, R. M., Álvarez Ojeda, V., \& Vélez Cedeño, J. G. (Enero-Junio de 2017). La gastronomía como atractivo turístico. Caso Pedernales, Ecuador. Retos Turísticos, 16(1), pp. 1-12. 